MATEC Web of Conferences 48, 02002 (2016)

DOI: $10.1051 /$ matecconf $/ 20164802002$

(C) Owned by the authors, published by EDP Sciences, 2016

\title{
Sensitivity improvement techniques for micromechanical vibrating accelerometers
}

\author{
Sergey Vtorushin ${ }^{1, a}$ and Tamara Nesterenko ${ }^{1}$ \\ ${ }^{1}$ National Research Tomsk Polytechnic University, 634050 Tomsk, Russia
}

\begin{abstract}
The paper presents the problems of detecting a desired signal generated by micromechanical vibrating accelerometer. Three detection methods, namely frequency, amplitude and phase are considered in this paper. These methods are used in micromechanical vibrating accelerometers that incorporate a force sensitive element which transforms measured acceleration into the output signal. Investigations are carried out using the ANSYS finite element program and MATLAB/Simulink support package. Investigation results include the comparative analysis of the output signal characteristics obtained by the different detection methods.
\end{abstract}

\section{Introduction}

Micromechanical vibrating accelerometers (MMVA) have been widely spread rather recently. Much attention is paid to these accelerometers due to their specific properties and operational characteristics that essentially differ from that of micromechanical capacitive accelerometers (MMCA) used today. The MMVA advantages include the high sensitivity that exceeds that of traditional capacitive accelerometers by one order more; high dynamic range and stability [1].

Presently, the microelectromechanical systems (MEMS) are manufactured by leading companies of the USA, Japan, Korea, China, and other countries. The Draper Laboratory is an American not-forprofit research and development organization that one of the first started the investigations of silicon MMCA. Accelerometers designed and produced by the Draper Laboratory possess the stability of scaling factor of not less than $1 \mathrm{ppm}$ [2]. MMVA designed in the University of California, Berkeley, possesses the sensitivity of $17 \mathrm{~Hz} / \mathrm{g}$. This accelerometer incorporates two oscillators that provide the decrease of its thermal sensitivity [3]. Researchers from Seoul National University (South Korea) designed the micromechanical resonator silicon accelerometer having $70 \mathrm{~Hz} / \mathrm{g}$ sensitivity at $12 \mathrm{kHz}$ resonance frequency of oscillators [4]. Chinese MEMS developers are currently involved in modeling silicon accelerators. Thus, for example, Tsinghua University (Peking) in cooperation with the Institute of Microelectronics developed the MMVA prototype having $27,3 \mathrm{~Hz} / \mathrm{g}$ sensitivity and $167,8 \mu \mathrm{g}$ resolution [5]. The past decade has seen remarkable advances in the development of MEMS technologies owing to their high reliability and low costs [6].

\footnotetext{
${ }^{a}$ Corresponding author : vtorushin1991@mail.ru
} 


\section{Operation principle of single-axis MMVA}

The principle of operation of any micromechanical vibrating accelerometer incorporating a sensitive element (oscillatory-type transducer), is based on the difference between the transducer eigenfrequencies induced by its stess-strain state. A force sensitive element or silicon resonator shown in Figure 1 can be used as an oscillatory-type transducer.

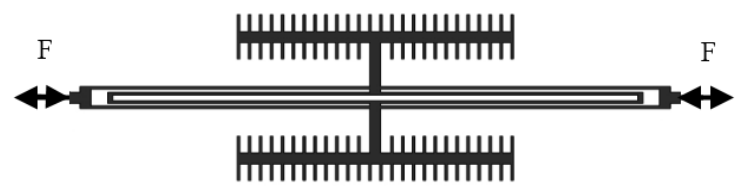

Figure 1. Force sensitive element.

Under forces $F$, the resonator eigenfrequency is changed depending on the force direction. The resonator eigenfrequency can vary not only under longitudinal and transverse forces but also force moments. At the same time, the maximum eigenfrequency unbalance is observed under the longitudinal forces [7].

The change in resonator eigenfrequency induced either tensile or compressive forces can be calculated from

$$
\Delta f=i^{2} \pi^{2} \sqrt{\frac{E I_{x}}{m l^{4}}} \sqrt{1 \pm \frac{\Delta F l^{2}}{i^{2} \pi^{2} E I_{x}}}
$$

In (1), the first three multipliers describe the eigenfrequency mode $i$ without considering the prestressed state of resonator. The last multiplier describes the longitudinal force $F$ the positive sign of which corresponds to tensile force, while the negative - to compressive. The changes in eigenfrequency and longitudinal force are proportional to each other.

This principle can be used to design a sensitive single axis vibratory accelerometer shown in Figure 2.

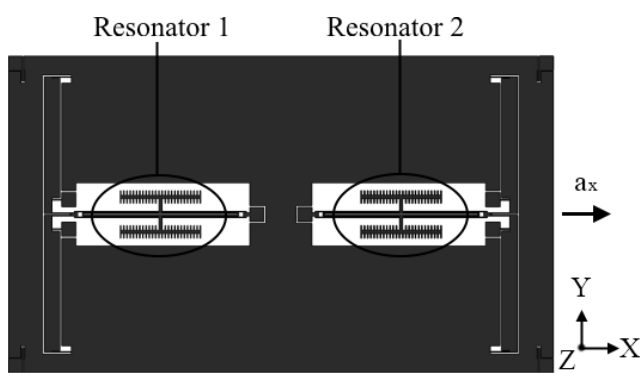

Figure 2. Schematic structure of micromechanical vibrating accelerometer with sensitive single axis.

The vibratory micromechanical accelerometer is provided with the inertial mass connected with the frame by the elastic wires and by the lever system with resonator. Under the external acceleration ax, the inertial mass is subjected to inertial forces transmitted by the lever system to silicon resonators. The lever system provides several times increase of the transmitted force depending on the arm. Internal stresses occurred in resonators produce changes in their eigenfrequencies, resonator 2 being extended and resonator 1 being compressed. The eigenfrequency shifting reflects the amount of the actual acceleration.

Thus, the lever system improves the sensitivity of accelerator to the acceleration measured, while the differential resonator connection circuit allows constructing the two-channel acceleration transforming circuit with the common-mode algorithmic compensation of thermal noise. 


\section{The model of single-axis MMVA}

The ANSYS domain modal analysis allows obtaining two working vibration modes of the accelerometer (Figure 3).

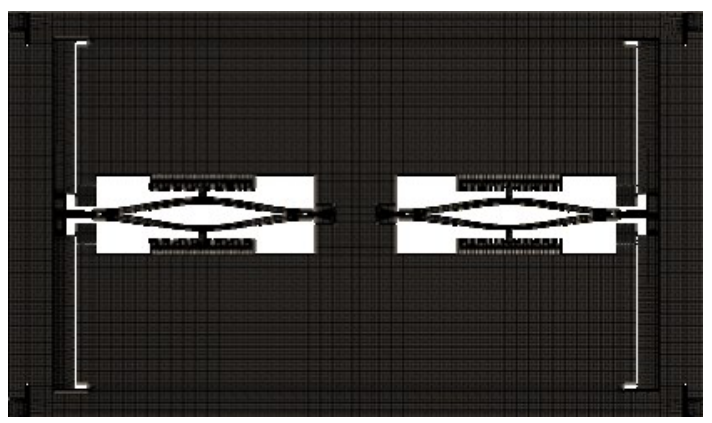

Figure 3. Schematic structure of micromechanical vibrating accelerometer with the fourth frequency mode.

Vibration excitations of silicon resonators are observed at the fourth frequency mode. Each couple of strings of the both resonators is excited in antiphase, thereby compensating the forces and force moments generated by each string and decreasing the energy out feed in the environment through the supports.

The two-channel differential resonator connection circuit increases twice the accelerometer sensitivity. According to (1), the eigenfrequency of resonator subjected to tensile force increases proportionally to the force $F$. The eigenfrequency of the second resonator decreases due to the compressive force. The total change in eigenfrequiency is calculated using the following equation:

$$
\Delta f_{\Sigma}=i^{2} \pi^{2} \sqrt{\frac{E I_{x}}{m l^{4}}}\left(\sqrt{1+\frac{\Delta F l^{2}}{i^{2} \pi^{2} E I_{x}}}-\sqrt{1-\frac{\Delta F l^{2}}{i^{2} \pi^{2} E I_{x}}}\right)
$$

As a result, we obtain the doubled change of the eigenfrequency. According to (2), the accelerometer sensitivity to longitudinal forces and, as consequence, measured acceleration can be improved when decreasing the flexural stiffness $E I_{x}$ of resonator strings and varying their length 1 .

In accordance with the ANSYS domain modal analysis accounting for pre-stressed state of resonator, the output characteristics are ascertained for the accelerometer. Figures 4 and 5 contain plots of these characteristics.

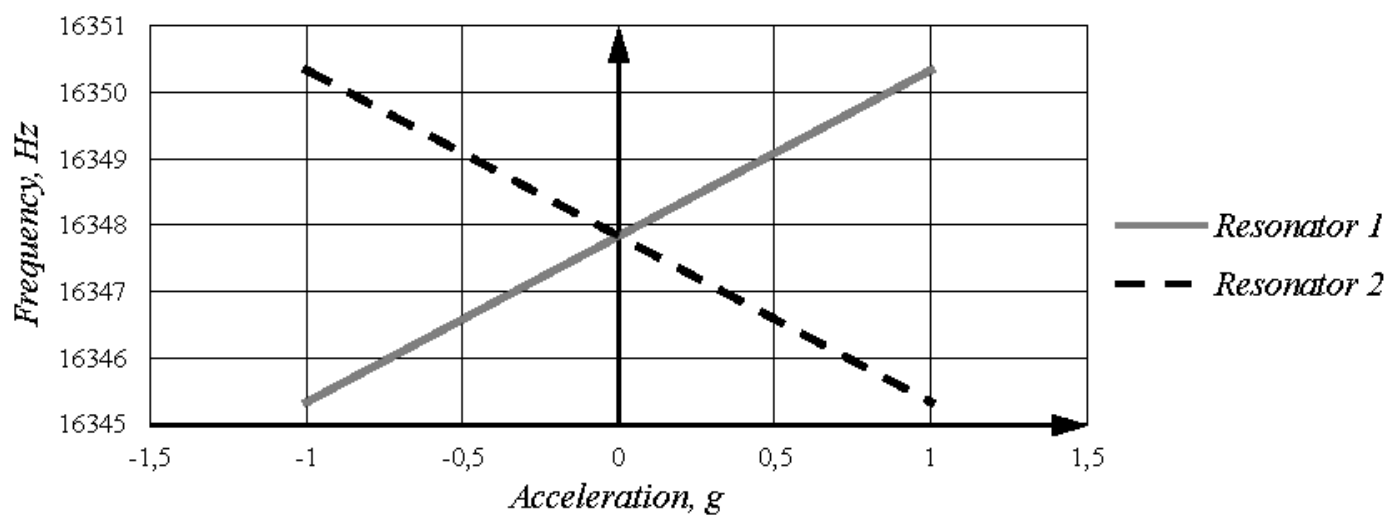

Figure 4. Output characteristic under $X$-axis acceleration. 


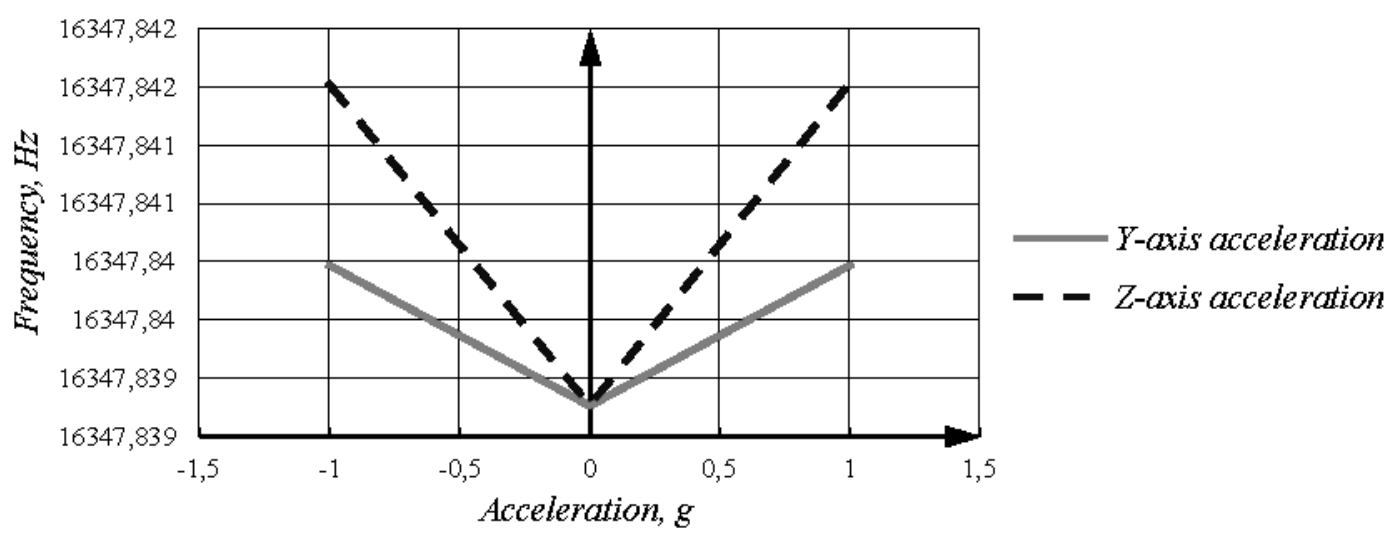

Figure 5. Output characteristic under $X$ - and $Y$-axis acceleration.

Affected by the $X$ - and $Y$-axis acceleration, eigenfrequencies are changed insignificantly only in one direction and can be completely compensated meeting the following conditions:

$$
\Delta f_{\Sigma}=i^{2} \pi^{2} \sqrt{\frac{E I_{x}}{m l^{4}}}\left(\sqrt{1+\frac{\Delta F l^{2}}{i^{2} \pi^{2} E I_{x}}}-\sqrt{1+\frac{\Delta F l^{2}}{i^{2} \pi^{2} E I_{x}}}\right)
$$

Herewith, the frequency difference under $X$-axis acceleration equals to $5,014 \mathrm{~Hz}$ in conformity with Figure 4 and equation (2).

\subsection{Amplitude and phase detection technique}

The amplitude and phase detection technique involves the measurement of the vibrational amplitude shift induced by acceleration. The maximum vibrational amplitude is observed at the coincidence of eigenfrequency of resonators and the excitation frequency of generator. The vibrational amplitude decreases at the eigenfrequency shift of resonators due to their stress-strain state. Depending on the mode of excitation, the operating point can be observed in the maximum peak of the amplitudefrequency curve or shifted both to left and right relative to this maximum. While selecting the mode of frequency mistuning with the operating point shift, it is possible to develop the differential measurement circuit.

The phase detection technique employs measurement of the phase difference of the reference-input signal produced by generator and vibrations produced by resonators. The phase difference at resonance is $90^{\circ}$. The eigenfrequency shift of resonators results in a change of the phase difference.

The mathematical simulation is used to calculate the accelerometer dynamics in order to evaluate each method by the maximum sensitivity criterion. The mathematical model presents the system of non homogeneous differential equations having the form

$$
m_{i} \ddot{y}+\frac{\omega_{i}(q) m_{i}}{Q} \dot{y}+\omega_{i}^{2}(q) y=F_{e} \sin (\omega t)
$$

This equation is written in modal coordinates and complies with the typical equation of the second-order vibratory link. The transfer function for each string takes the form

$$
W(s)=\frac{3.798 \cdot 10^{17}}{s^{2}+1027 s+1.055 \cdot 10^{10}}
$$


The amplitude- and phase-frequency curves of accelerometer are presented in Figure 6. The Qfactor is accepted to be 1000. The two curves describe the systems with ( $\mathrm{a}=1 \mathrm{~g})$ and without acceleration $(\mathrm{a}=0 \mathrm{~g})$.

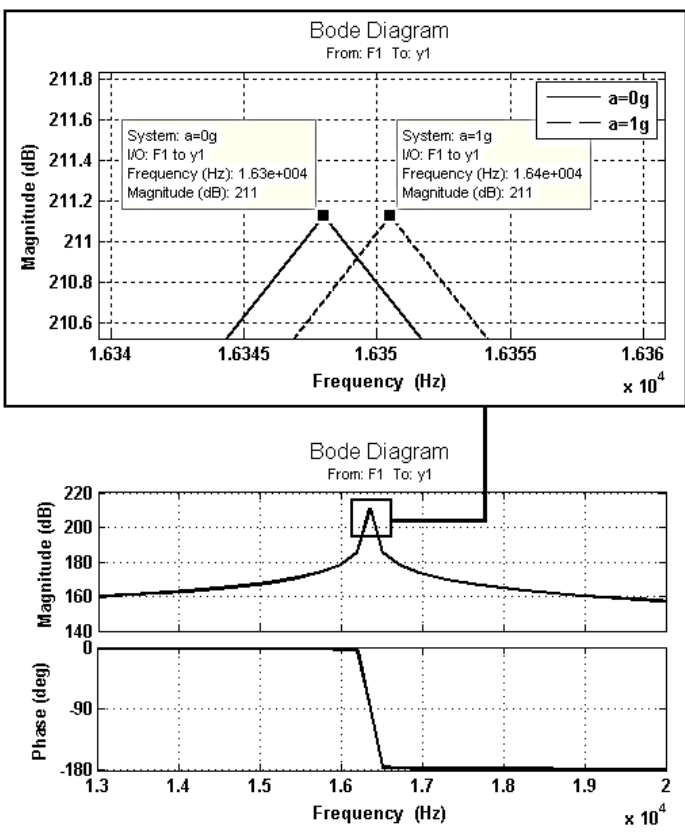

Figure 6. Amplitude- and phase-frequency curves for one string of resonator.

In Figure 7, the comparative characteristics of accelerometer are presented within the $0-1 \mathrm{~g}$ interval in accordance with the given frequency responses.

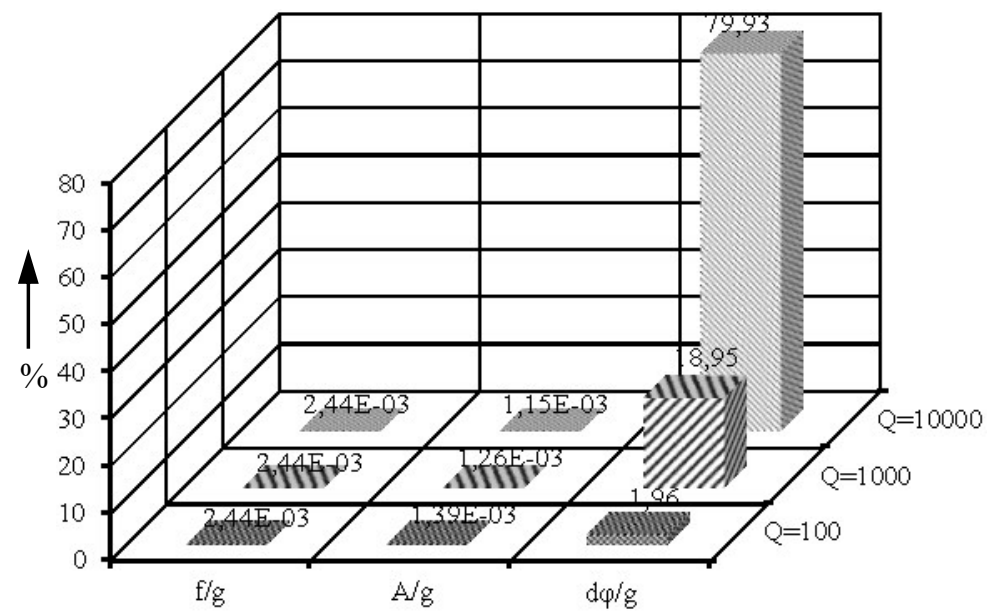

Figure 7. Comparative characteristics of accelerometer.

The maximum change is observed in the phase difference. The required frequency properties and $\Delta \varphi=71,9 \mathrm{deg} / \mathrm{g}$ sensitivity can be obtained for the accelerometer at $\mathrm{Q}=10000$ achieved by the pressure decrease in the frame. The changes in frequency and amplitude under the acceleration less depends on the Q-factor and equal to $\Delta \mathrm{A}=0,00266 \mathrm{~dB} / \mathrm{g}$ and $\Delta \mathrm{f}=5,014 \mathrm{~Hz} / \mathrm{g}$, respectively. 
These investigations showed that the maximum informativeness and sensitivity were achieved for the accelerometer with phase detection of a desired signal. The method of phase detection provides the design of accelerometer with the digital output, thereby considerably increasing its noise resistance.

\section{Conclusion}

These investigations showed that the maximum informativeness and sensitivity were achieved for the accelerometer with phase detection of a desired signal. The method of phase detection provides the design of accelerometer with the digital output, thereby considerably increasing its noise resistance.

\section{Acknowledgement}

This work was financially supported by the Ministry of Education and Science of the Russian Federation (agreement N 14.575.21.0068, unique identifier RFMEFI57514X0068).

\section{References}

1. D. W. Burns, R. D. Homing, W. R. Herb, J. D. Zook, H. Guckel, Sens. Actuator 53, 249 (1996)

2. L. Huang, H. Yang, Y. Gao, L. Zhao, J. Liang, Sensors 13, 15785 (2013)

3. A. A. Seshia, M. Palaniapan, T. A. Roessig, R. T. Howe, R. W. Gooch, T. R. Schimert, and S. Montague, Journal of microelectromechanical Systems 11, 784 (2002)

4. H. C. Kim, S. Seok, I. Kim, S.-D. Choi, K. Chun, Proceedings of the 13th International Conference on Solid-state Sensors, Actuators and Microsystems 1, 172 (2005)

5. Y. Jia, Y. Hao, R. Zhang, Chin. J. Semicod 2, 281 (2005)

6. T. G. Nesterenko, S. E. Vtorushin, E. S. Barbin and A. N. Koleda, IOP Conference Series: Materials Science and Engineering 81 (2015)

7. S. E. Vtorushin, T. G. Nesterenko, Fundamental research 2, 2553 (2015) 